\title{
Beautiful machines
}

\author{
Physicists are now, as ever, pushing the development of computing technologies. But they're also \\ innovating ways of using them.
}

You'd be hard pressed to argue for the existence of the computer as we know it, were it not for a group of physicists by the names of Walter Brattain, John Bardeen and William Shockley. Tasked with replacing the high-maintenance vacuum tubes used at Bell Labs in the 1940s, the team succeeded in creating a solid-state amplifier - building the foundations for modern electronics.

The path from the humble beginnings of the world's first electronic digital computer - crafted in a basement lab by another physicist duo, John Vincent Atanasoff and Clifford Berry - to the device paradise in which we now live, is paved with advances made by countless physicists. But it's a two-way street: modern physics research is also indebted to the power afforded by these machines. And as a result, physicists are often deeply involved in devising ways of putting the new toys to good use.

Part of this drive is born of sheer necessity. Research in physics is now heavily reliant on numerical simulations that, together with the feats of data management they require, can demand remarkable computing power. But large-scale scientific endeavours have historically been linked to significant computing advances. In the early post-war years, the first electronic computer to be deployed at Los Alamos, the MANIAC, quickly became irresistible for many physicists. Enrico Fermi, for example, immediately put it to work to implement his algorithm for the pion-proton phase shift calculation (pictured).

Another famous case in point was the brainchild of Tim Berners-Lee, the physics graduate who created the World Wide Web. Tasked with meeting the demands of massive information exchange between the thousands of physicists working at CERN and their collaborators in other parts of the world, Berners-Lee came up with a fix - and in doing so, changed the course of history.

In this month's issue of Nature Physics, we showcase some of the more recent developments in the way that physicists are approaching — and advancing — the world of computing. We've asked four experts to weigh in with a series of opinion pieces designed to highlight topics at this frontier in our Focus on computing in physics.

Following in Berners-Lee's footsteps, scientists at CERN are again leading the

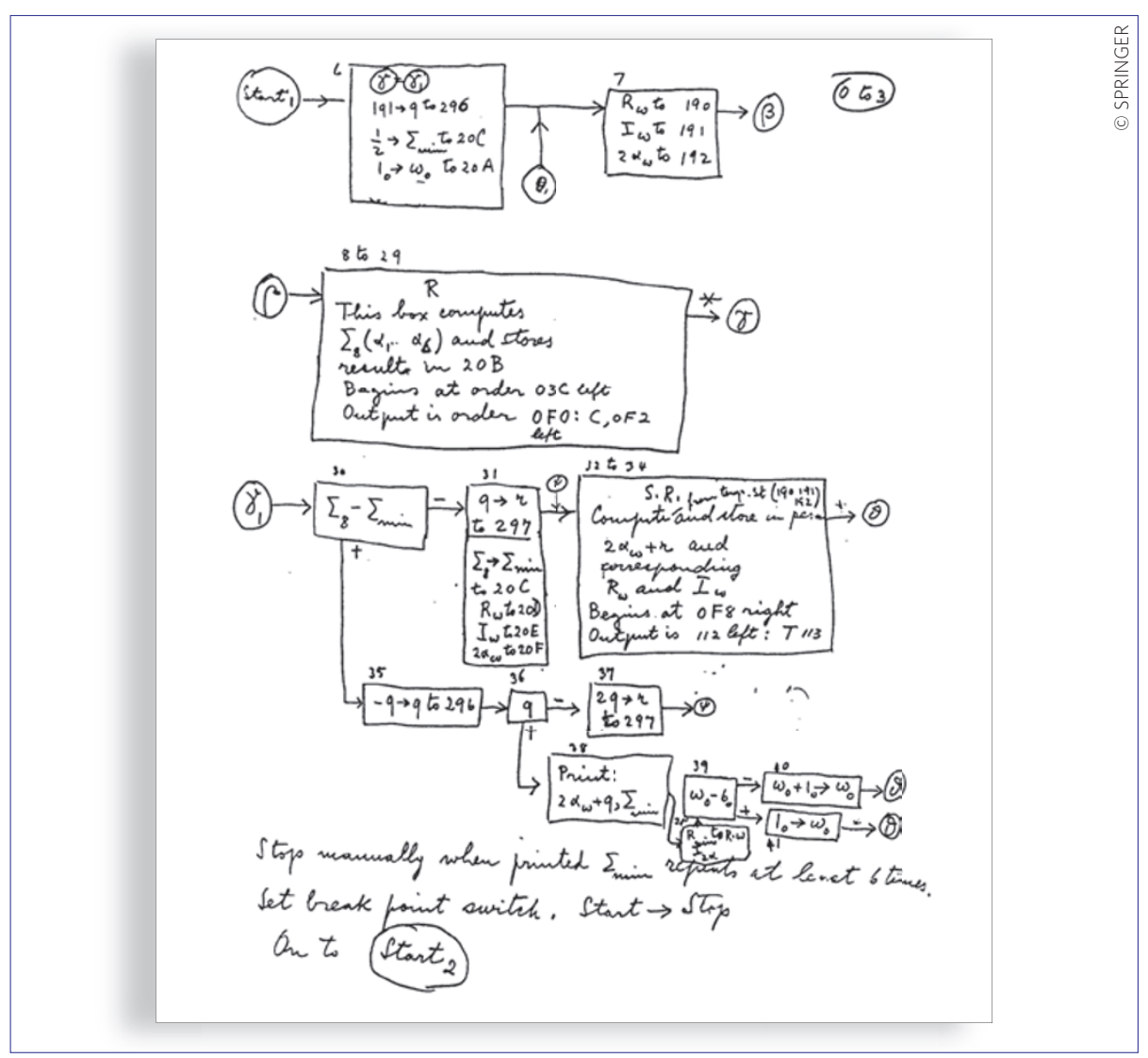

charge into new territory by exploring the possibilities of cloud computing to handle their enormous data production. On page 373, Sergey Panitkin gives us an insider's take on the progress that the ATLAS collaboration is making, using in-house and commercial cloud services, as well as other high-performance computing solutions.

Cloud providers aren't the only commercial entities changing the face of computational physics. With the introduction of graphics processing units (GPUs) to accelerate computing, graphics-card manufacturers, like Nvidia, are also becoming direct sponsors of innovative new research in the physical sciences. On page 369, Thomas Schulthess examines the way that physicists and physics research are evolving to make use of this rapidly developing hardware, characterized by increasing complexity. The answer, he argues, lies in keen integration of multidisciplinary techniques.

In this respect, developing a more open dialogue between scientists would seem essential. Tony Hey and Mike Payne explore the challenges that physicists face in the push towards open research on page 367 of this issue. They argue that scientific reproducibility hinges on widespread code sharing throughout the academic community. Crucially, they note that the quality of the programming is also key to ensuring that scientific research remains effective and reproducible.

The future of computing looks bright, and it's moving quickly. According to the claims made at Nvidia's recent GPU Technology Conference (http://www.gputechconf.com/), their GeForce Titan X will boast a whopping seven teraflops, making it a supercomputer in itself. For comparison, the Cray- 1 achieved 80 megaflops in 1982, and in 2013, the state of the art ran at just under 34 petaflops. The world of research will soon have unparalleled computing power at its fingertips. It remains to be seen what innovation the physics community will magic up next, in response to this development. 